\title{
The new theory of gravitation representing the movement of planets
}

\author{
Tomasz Borowski \\ Astronomical Observatory 22 m. 12 Mickiewicza Street, woj. zachodniopomorskie \\ 78-520 Zlocieniec, Poland \\ E-mail address: tomasz.elvis.borowski@wp.pl
}

\begin{abstract}
In this paper, a theory of the phenomenon of planetary circulation around the Sun is presented, as well as the stability of solar systems is explained. This theory describes the circulation of planets and other mass-holding bodies around the Sun as a phenomenon consisting in the principle of differential pressure in solar systems and thus excludes gravitation phenomenon as the phenomenon of attraction.
\end{abstract}

\section{Keywords}

dark matter; gravitation; radiation; attraction; planetary systems

\section{INTRODUCTION}

Newtonian physics describes certain properties and relationships between matters [1, 2].

The Einstein's physics describes the properties of waves as well as relationships between wave and matter $[3,4]$.

The physics of gravitation is generally known and mathematically described $[5,6]$ but it does not explain the mechanism of attraction between two mass-holding bodies.

If we change the assumption that gravitation is not the phenomenon of attraction but of movement of a material body from a medium with higher pressure to that with lower pressure in the space thus this new definition of gravitation with changed assumption is logical and omnipresent both in the Solar system and the whole universe.

\section{THEORY OF THE PHENOMENON OF DIFFERENTIAL PRESSURE DEVELOPMENT IN THE SOLAR SYSTEM}

The factor joining the whole Solar system and galaxies all in one piece would have to have higher speed than the velocity of light.

At present, no higher speed is known than the velocity of light, thus the phenomenon ascribed to gravitation must have other form than that know currently as the phenomenon of gravitational pull.

At present, the phenomenon of gravitational pull occurs when two mass-holding objects are close to each other but it does not explain the mechanism of attraction. 
Since it is not possible to observe and explain the mechanism of gravitational pull, another physical phenomenon should be ascribed which would explain the movement of mass-holding objects.

Such a theoretical physical phenomenon is development of differential pressure between two mass-holding objects. As a result of astronomical observations, it has been already found long ago that smaller astronomical objects circulate around large ones (Venus - Sun, Moon Earth, etc.).

Therefore, it is possible to conclude that larger astronomical objects create higher negative pressure around themselves than smaller ones which also create negative pressure.

\subsection{The phenomenon of lower pressure development near mass-holding objects}

Every mass-holding matter emits energy outward. The energy emitted by a matter is absorbed by dark matter which does not hold material mass.

Dark matter lowers then its volume and therefore its pressure (Fig. 1). Such a phenomenon occurs near material objects (Sun, planets, etc.). Larger material objects emit larger quantities of energy and therefore dark matter is able to absorb larger quantities of energy emitted by a mass-holding matter. Thus, a larger area of negative pressure develops.

Matter and dark matter occur together but at different volumetric quantities. The larger cluster of mass-holding matter, the smaller is the volume of dark matter around the mass. In the Solar system, a differential pressure (difference in negative pressures) between the matter and the dark matter occurs. The closer to a matter cluster, the more increased are its volume and pressure and the same more decreased is the volume and therefore the pressure of dark matter.

This whole phenomenon connected with the decrease of dark matter pressure is being induced by absorption of radiation from the mass-holding matter.

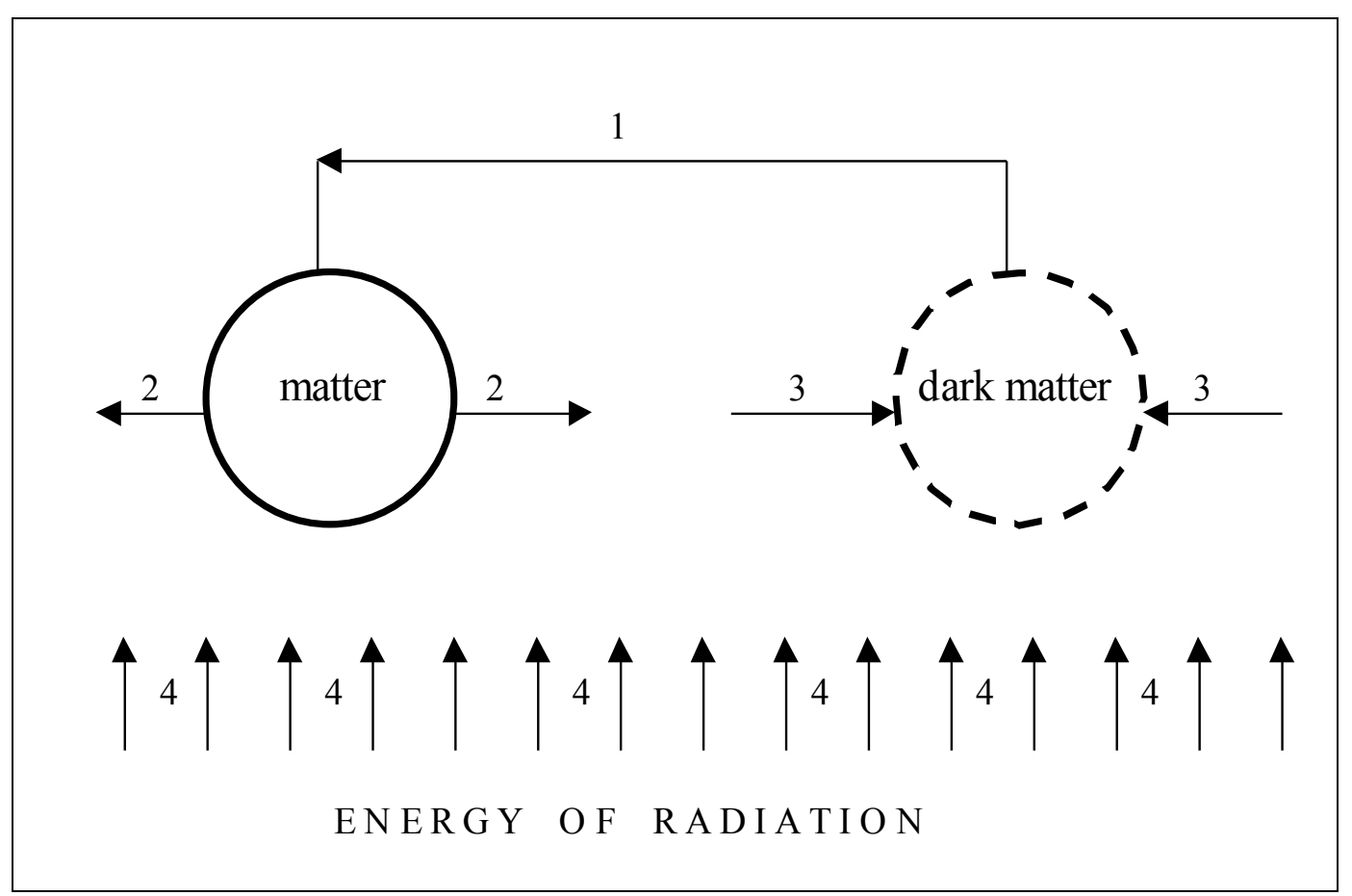

Fig. 1. The phenomenon presents development of lower pressure (negative pressure) nearthe matter. 
In Figure 1 and 3, there are numbered arrows representing as follows:

1- direction of the increase of matter volume (matter pressure increases) or the decrease of dark matter volume (dark matter pressure decreases),

2- the increase of matter volume (matter pressure increases) through energy absorption,

3- the decrease of antimatter volume (dark matter pressure decreases) through energy absorption,

4- direction of the energy of radiation.

\subsection{The phenomenon of higher pressure development in the cosmic space beyond the material system}

The mechanism of developing higher pressure of dark matter is connected with a smaller quantity of mass-holding matter which emits energy causing that the volume of dark matter, and the same its pressure, increases (Fig. 2).

Such a space is being found far away from mass-holding objects. These are spaces beyond the material systems where dark matter pressure is higher than near mass-holding objects. The highest pressure of dark matter occurs in the cosmic space beyond systems, e.g. galactic systems.

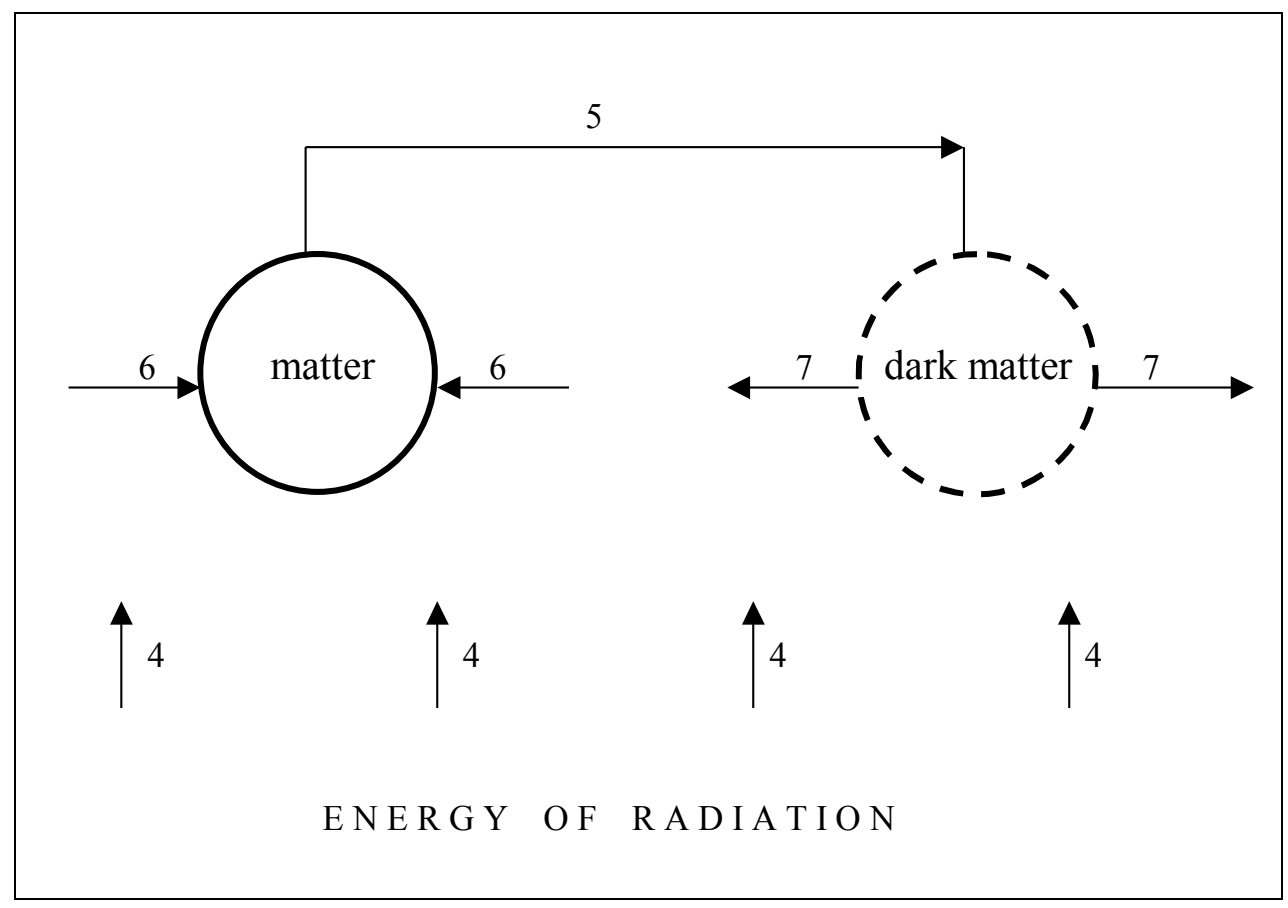

Fig. 2. The phenomenon presents deficiency of matter, i.e. deficit of radiation or its absence near the dark matter.

In Figure 2 and 3, there are numbered arrows representing as follows:

4- direction of the energy of radiation.

5- direction of the decrease of matter volume (matter pressure decreases) or the of dark matter volume (dark matter pressure increases),

6- the decrease of matter volume (matter pressure decreases) induced by deficit or absence of the absorption of radiation energy,

7- the increase of dark matter volume (dark matter pressure increases) through deficit or absence of the absorption of radiation energy coming from the matter. 


\subsection{The state of equilibrium between matter and dark matter}

In Figure 3 is presented a theoretical relationship of the equilibrium between massholding matter and dark matter which does not hold material mass. The mass-holding matter emits energy. This energy-emitting matter decreases its internal energy, i.e. decreases its volume and the same its pressure. The energy emitted from the matter is being absorbed by dark matter.

When absorbing the energy, the dark matter decreases its pressure and the same its volume. When emitting the absorbed energy, the dark matter increases its volume and the same its pressure. The energy emitted by dark matter is being absorbed by mass-holding matter. When absorbing the energy from dark matter, this matter increases its volume and pressure (Fig. 3).

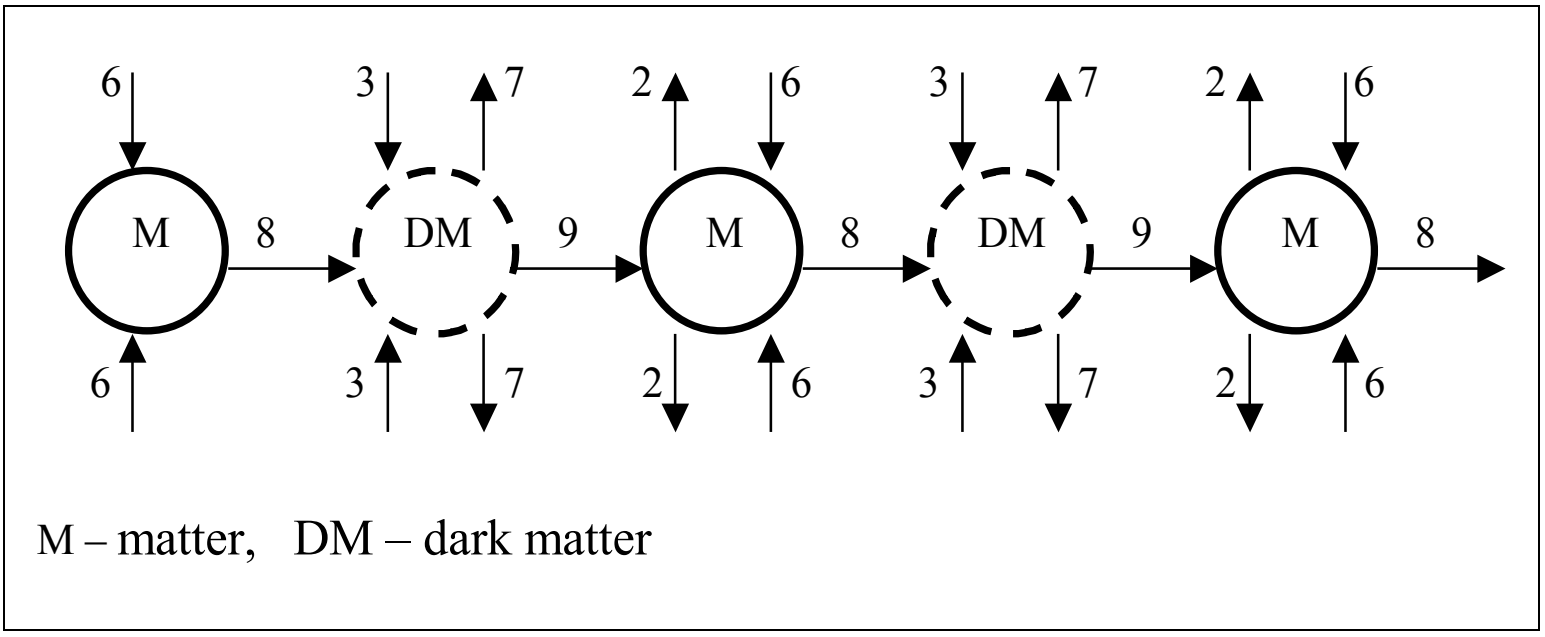

Fig. 3. The theoretical relationship of the equilibrium between mass-holding matter and dark matter which does not hold material mass.

In Figure 3, there are numbered arrows representing as follows:

$1,2,3,4,5,6,7-$ look figures 1 and 2,

8 - the matter emits energy,

9 - the dark matter emits energy.

\section{CONCLUSIONS}

Theory of the phenomenon of mass-holding objects movement around the Sun, as well as smaller material objects (e.g. Moon - Earth), is induced by the equilibrium of pressure between matter and dark matter.

This equilibrium is an equilibrium between the centrifugal force of orbiting planet and the increase of negative pressure inside the Solar system (e.g. Venus - Sun) or smaller planetary system (e.g. Moon - Earth). A change in this equilibrium will cause a planet to leave the Solar system or to be absorbed by a larger object, e.g. by the Sun.

After rethinking the theory of the phenomenon of orbital movement of planets, a new definition of gravitation is coming to mind: 


\section{Gravitation is the phenomenon of repulsion consisting in the movement of material body from a medium with higher pressure of dark matter to that with lower pressure of dark matter in the space.}

Presently discovered Higgs boson or Higgs boson-like particle possesses the same 'properties' as the dark matter does: they tie the universe. One may suggest the statement that the Higgs boson or Higgs boson-like particle and the dark matter are the same elements appearing in the universe under different names.

\section{REFERENCES}

[1] Jonathan Bain, Studies In History and Philosophy of Science Part B: Studies In History and Philosophy of Modern Physics, Vol 35, Issue 3 (2004) 345-376

[2] A.F. Steklain and P.S. Letelier, Physics Letters A, Vol 352, Issues 4-5, (2006) 398-403

[3] Lorenzo Sindoni, Phys. Rev. D 83 (2011) 024022

[4] Christian Sanner, Edward J. Su, Aviv Keshet, Wujie Huang, Jonathon Gillen, Ralf Gommers, and Wolfgang Ketterle, Phys. Rev. Lett. 106 (2011) 010402

[5] Carl Hoefer, Studies In History and Philosophy of Science Part A, Vol 25, Issue 3, (1994) 287-335

[6] John D. Norton, Studies In History and Philosophy of Science Part B: Studies In History and Philosophy of Modern Physics, Vol 37, Issue 1 (2006) 71-100 